\title{
A Low-Noise RF Voltage-Controlled Oscillator Using On-Chip High-Q Three-Dimensional Coil Inductor and Micromachined Variable Capacitor
}

\author{
Darrin J. Young, Vincent Malba*, Jia-Jiunn Ou, Anthony F. Bernhardt*, and Bernhard E. Boser \\ EECS Department, University of California, Berkeley, CA 94720 \\ * Lawrence Livermore National Laboratory
}

\begin{abstract}
A RF voltage-controlled oscillator (VCO) employs an onchip, high-Q, three-dimensional (3-D) coil inductor and micromachined variable capacitor for frequency tuning. Unlike conventional spiral inductors, the $3-\mathrm{D}$ inductor minimizes the substrate loss and achieves a record $Q$ of 30 at $1 \mathrm{GHz}$. The micromachined variable capacitor achieves a $15 \%$ tuning range with a nominal $2 \mathrm{pF}$ capacitance and a $\mathrm{Q}$ value above 60 at 1 $\mathrm{GHz}$. Both passive components are fabricated on silicon substrates and thus amenable to monolithic integration with standard IC processes. The prototype VCO exhibits a phase-noise of $-136 \mathrm{dBc} / \mathrm{Hz}$ at $3 \mathrm{MHz}$ offset frequency from the carrier, suitable for most wireless communication applications, in particular GSM [1]. The VCO is tunable from $855 \mathrm{MHz}$ to $863 \mathrm{MHz}$ under $3 \mathrm{~V}$, limited by the test setup.
\end{abstract}

\section{INTRODUCTION}

Increased demand for wireless communication motivates a growing interest in monolithic personal communication transceivers [2]. Current radio designs, however, depend on off-chip components to implement key building blocks such as the lownoise RF VCOs. The off-chip devices increase package complexity, final system area, and cost. Therefore, monolithic implementations are highly desirable.

The various cellular telephony standards require VCOs with frequencies in the low Gigahertz range and a tuning range less than $5 \%$ of the carrier frequency. Narrow channel spacing and large blocking signals call for an extremely low phase-noise from the oscillator. Phase-noise below $-135 \mathrm{dBc} / \mathrm{Hz}$ at $3 \mathrm{MHz}$ offset frequency, for example, is required for GSM [1].

Current VCO designs in personal communication transceivers employ an off-chip high-Q LC tank circuit to meet the low phase-noise requirement. Typical values are on the order of $5 \mathrm{nH}$ with a $\mathrm{Q}$ of 30 for the inductor, and $2 \mathrm{pF}$ with a $\mathrm{Q}$ of at least 50 for the varactor. Frequency tuning is achieved by modulating the depletion width of the varactor diode. A typical capacitance change of at least $10 \%$ is required to cover the tuning range. However, the off-chip components rely on processes and materials that differ substantially from standard IC fabrication and are consequently not compatible for monolithic integration.

On-chip silicon junction capacitors and spiral inductors have also been used to implement monolithic VCOs. However, this approach results in a poor phase-noise because of the low $\mathrm{Q}$ passive components $[3,4]$. The silicon junction diodes exhibit an excessive series loss resulting in a limited $Q$ value below 10 . The on-chip spiral inductors suffer from an even lower $Q$ around 3 at $1 \mathrm{GHz}$ [5]. Two issues contribute to this low quality factor: eddy currents in the substrate and metal resistive losses. The first problem can be addressed in part by removing the silicon substrate underneath the inductor, leading to a $Q$ value around 5 [6]. Reference [7] reports a $Q$ close to 10 at $1 \mathrm{GHz}$ by using copper traces on sapphire substrate. While improving the $Q$ value, sapphire substrates are incompatible with standard IC processes.

In this paper, we report a low phase-noise RF VCO using a silicon IC-compatible, high-Q, 3-D coil inductor [8] and micromachined variable capacitor [9]. The oscillator achieves a low phase-noise suitable for most wireless communication applications. To reduce the fabrication complexity of the prototype oscillator, the inductor, variable capacitor, and active electronics are realized on separate substrates and wire bonded to form the VCO. Because all the components are fabricated on silicon wafers, they are amenable to integration on the same substrate.

\section{MONOLITHIC 3-D COIL INDUCTOR}

The 3-D high-Q coil inductor, shown in Figure 1, is one of the key elements to achieve a low phase-noise in the VCO. The device consists of two turns of $5 \mu \mathrm{m}$-thick, $50 \mu \mathrm{m}$-wide copper traces electroplated around an alumina core. Compared to spiral inductors, this geometry minimizes the coil area in close proximity to the substrate and hence the eddy current loss, which is the dominant contributor to the limited $\mathrm{Q}$ value of spiral designs. The proposed device thus achieves a maximized $\mathrm{Q}$ and self-resonant frequency. Copper is selected as the interconnect metal for its low sheet resistance, critical for achieving a high Q. Alumina is used as the core material because of its negligible loss tangent at high frequencies, another key parameter to ensure a high $\mathrm{Q}$ value.

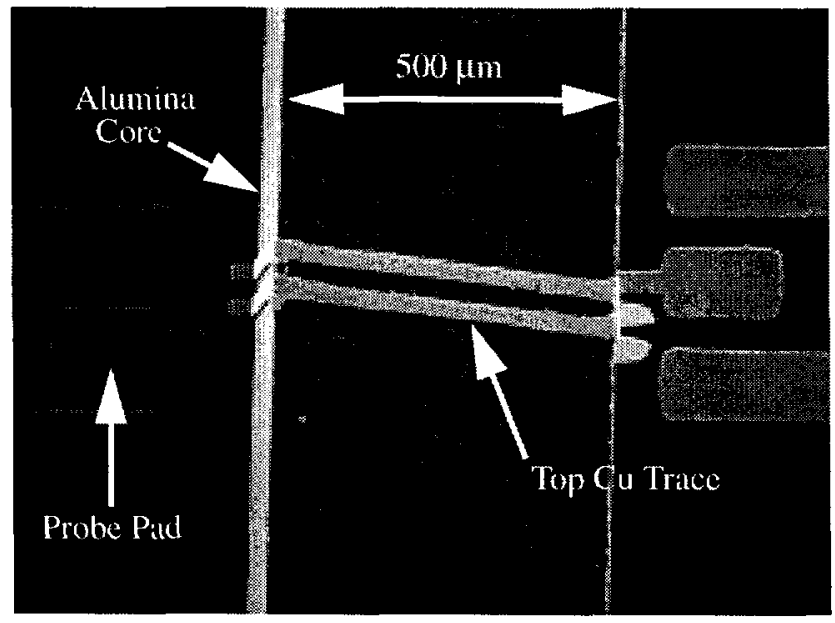

(1a) Top View 


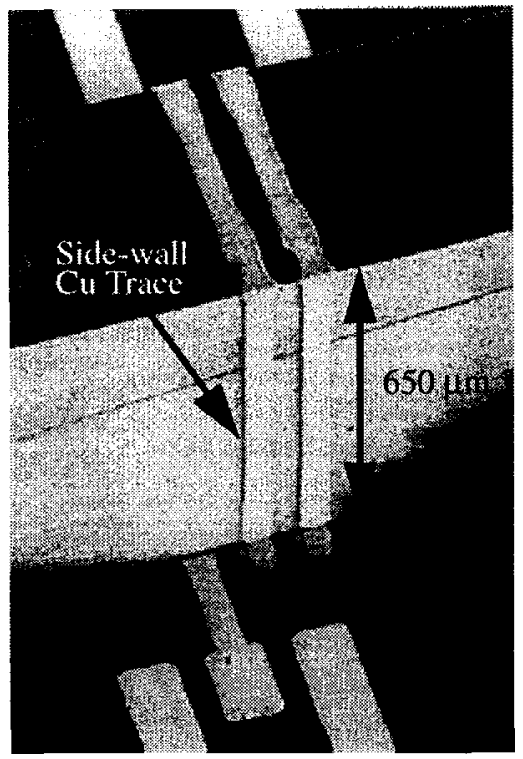

(1b) Side View

Figure 1. SEM of a Two-Turn 3-D Coil Inductor

The fabrication process is described in reference [8] in details. Figure 2 illustrates the simplified process flow. A $5 \mu \mathrm{m}$ thick low-temperature oxide is deposited first to serve as an insulating layer (Fig. 2a). Electroplated copper with $5 \mu \mathrm{m}$ thickness forms the inductor bottom metal traces (Fig. 2b-2c). The alumina core is then attached to the bottom traces with an adhesive film and covered by sputtered copper seed layer with electroplated photoresist on top (Fig. 2d). To fabricate the copper traces on the side-wall and the top of the core, the resist is exposed with a three-dimensional maskless direct-write laser lithography tool. After developing the resist, $5 \mu \mathrm{m}$ thick copper traces are electroplated around the core to complete the inductor coil (Fig. 2e). Because of the low processing temperature (170 ${ }^{\circ} \mathrm{C}$ maximum), the inductors can be fabricated on top of wafers with completed electronics without affecting the characteristics of active devices. This is particularly crucial in RF applications where the availability of the most recent IC technology is a critical competitive advantage.

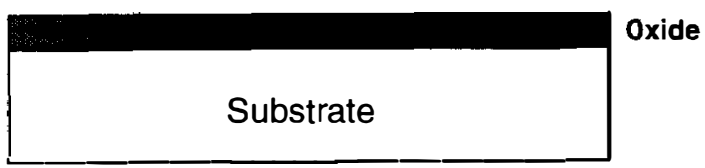

(2a)

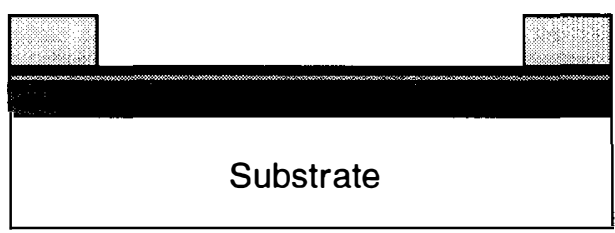

Photo-resist Curri Seed Oxide

(2b)

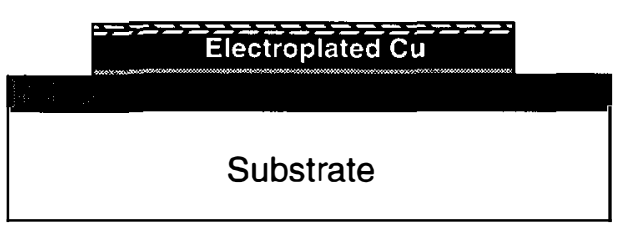

Au/Ni

Cu/Ti Seed Oxide

(2c)

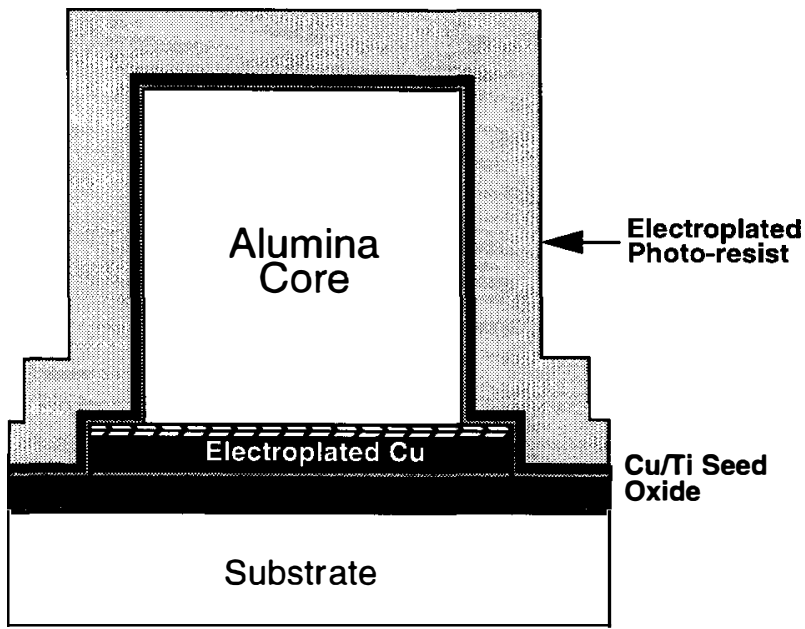

(2d)

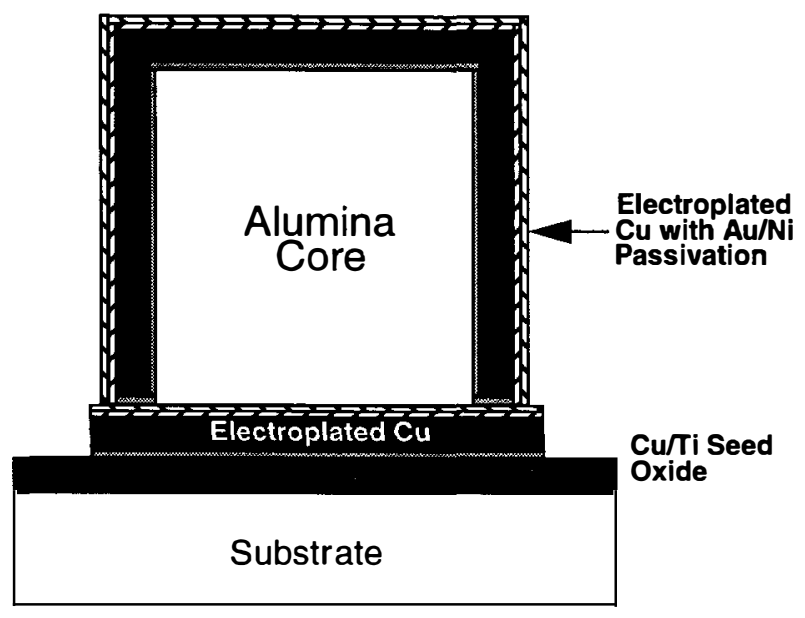

(2e)

Figure 2. 3-D Inductor Fabrication Process Flow

The fabricated two-tum inductor achieves $8.2 \mathrm{nH}$ inductance with a measured $Q$ of 16 at $1 \mathrm{GHz}$, as shown in Figure 3.

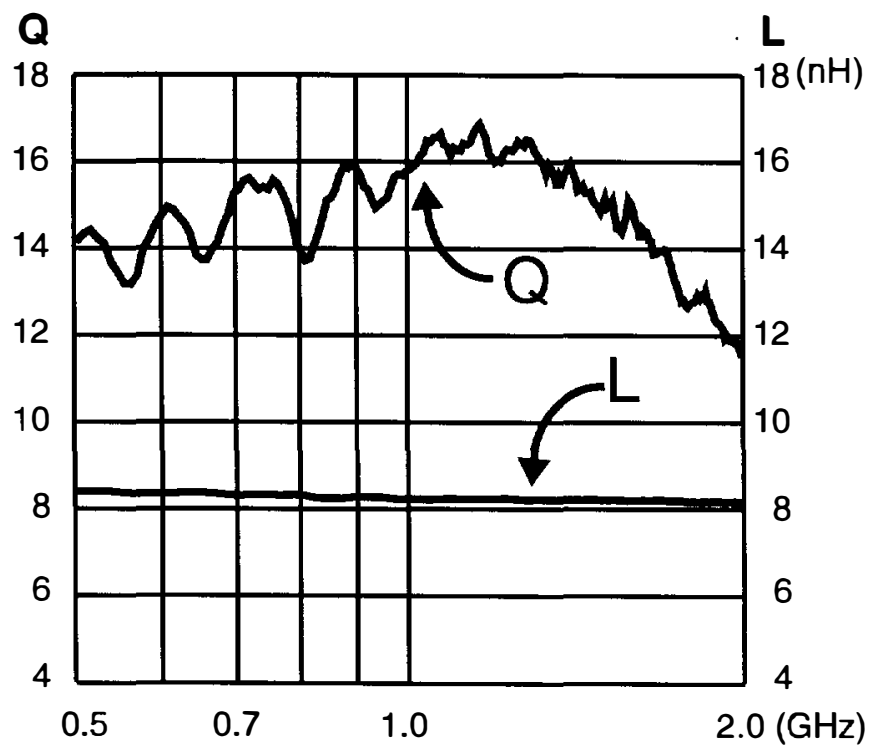

Figure 3. L and $\mathrm{Q}$ vs. Frequency for a Two-Tum Inductor 
This performance is substantially superior to that of spiral inductors with typical $\mathrm{Q}$ values around 3. A one-turn inductor demonstrates a record Q of 30 measured at $1 \mathrm{GHz}$ with $4.8 \mathrm{nH}$ inductance, which matches the quality factor of discrete counterparts. The self-resonant frequencies for these devices are over $8 \mathrm{GHz}$, well above the frequency of interest.

\section{MICROMACHINED VARIABLE CAPACITOR}

The high-Q variable capacitor is another key element in the low phase-noise VCO. This is realized as a surface-micromachined all-aluminum microstructure [9]. Figure 4 presents an SEM of a fabricated single capacitor. It consists of a thin aluminum plate suspended in air nominally $1.5 \mu \mathrm{m}$ above a bottom aluminum layer by four mechanical springs. Aluminum is selected as the structural material for its low sheet resistance, critical for achieving a high $\mathrm{Q}$ value.

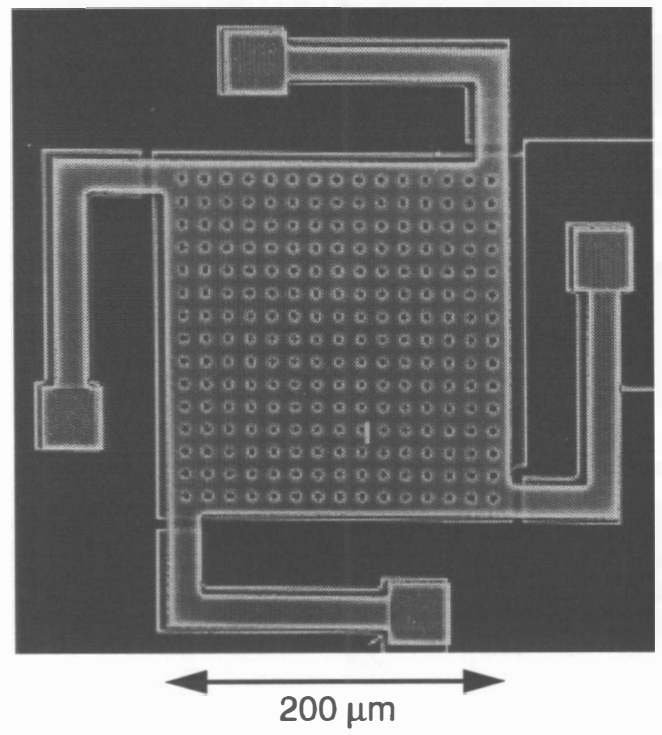

Figure 4. SEM of a Micromachined Variable Capacitor

A DC bias applied across the capacitor causes an electrostatic pull-down force and consequent reduction of the air gap, resulting in a capacitance increase. Figure 5 shows four such capacitors connected in parallel with $2.04 \mathrm{pF}$ at zero bias and $2.35 \mathrm{pF}$ at $3 \mathrm{~V}$, corresponding to a $15 \%$ capacitance increase. The variable capacitor achieves a measured Q over 60 at $1 \mathrm{GHz}$. This matches or exceeds the quality factor of discrete varactor diodes, and is at least an order of magnitude better than that of a typical on-chip silicon junction capacitor.

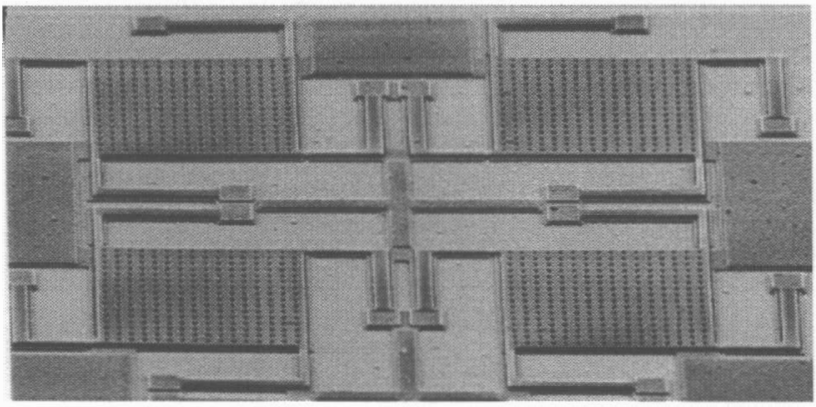

Figure 5. Four Parallel-Connected Micromachined Capacitors
The fabrication technology is fully compatible with standard IC processing [9], permitting the capacitor to be fabricated on top of wafers with completed electronics. Ultimately, the micromachined capacitors could be realized with copper, permitting co-fabrication with the inductors. It is expected that the low intrinsic stress of electroplated copper [10] would also reduce the warping, thus allowing a smaller air gap. This would result in a reduced area and tuning voltage.

\section{PROTOTYPE VCO AND MEASUREMENTS}

The high-Q passive components and a CMOS die containing active electronics are attached to a test board and wire bonded to form the VCO, as shown in Figure 6.

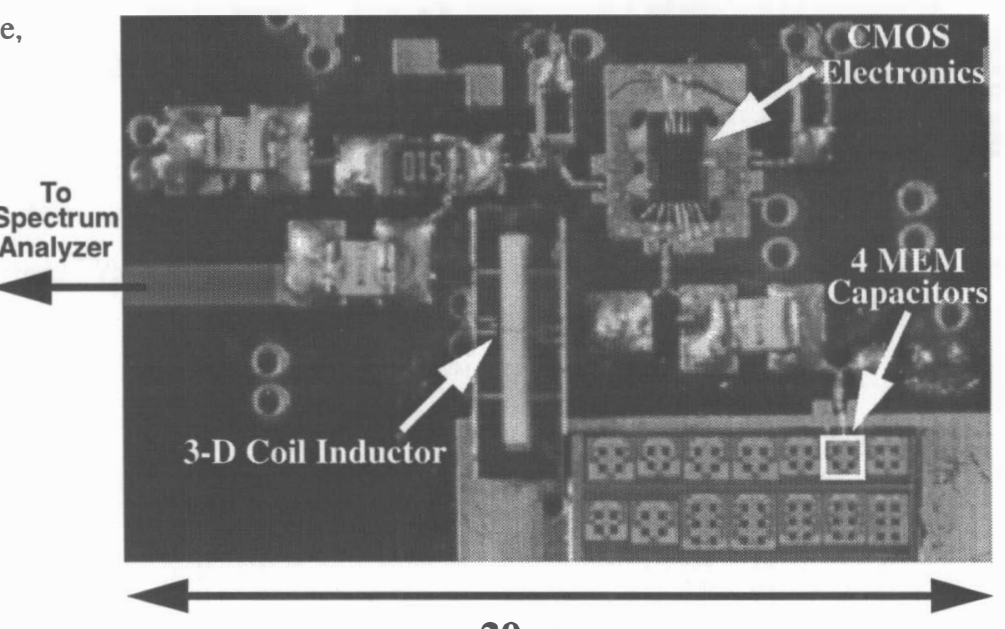

$20 \mathrm{~mm}$

Figure 6. Prototype VCO Test Board

Figure7 illustrates the VCO configuration. The Colpitt's oscillator topology is chosen for its simplicity. The electronics are fabricated with HP's $0.8 \mu \mathrm{m}$ CMOS process.

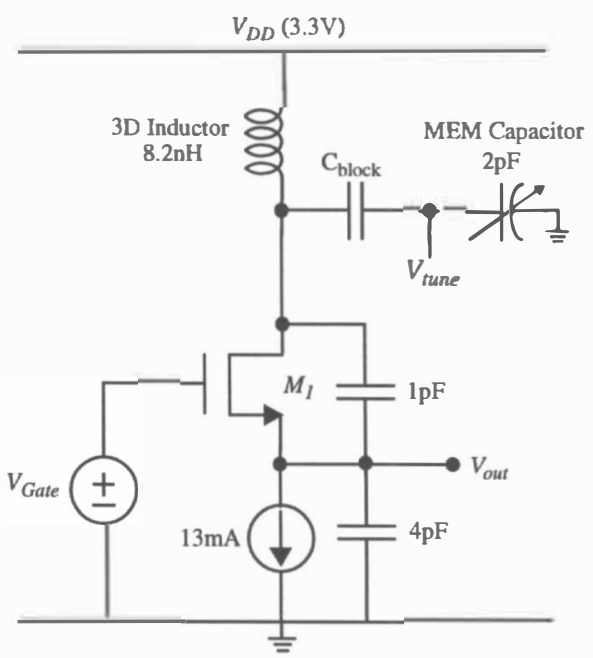

Figure 7. VCO architecture

Figure 8 shows the oscillator output power spectrum at $863 \mathrm{MHz}$ with a phase-noise of $-136 \mathrm{dBc} / \mathrm{Hz}$ measured at $3 \mathrm{MHz}$ offset frequency, as plotted in Figure 9. 


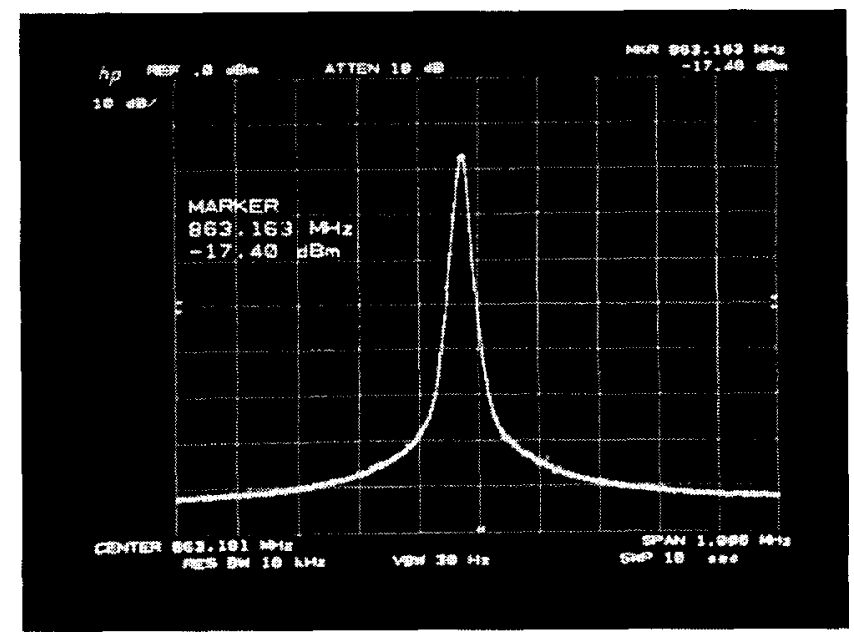

Figure 8. VCO Output Power Spectrum

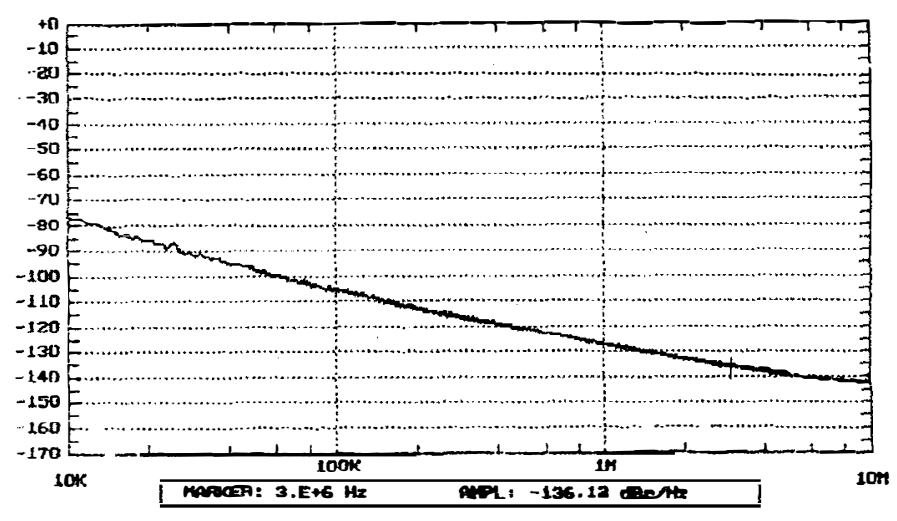

Figure 9. Measured Phase-Noise Plot

This performance is suitable for most wireless communication applications, in particular GSM. This level of performance has not been achieved by VCOs relying on on-chip spiral inductors and silicon junction capacitors. The prototype oscillator dissipates $43 \mathrm{~mW}$ from a $3.3 \mathrm{~V}$ supply, and is tunable from $855 \mathrm{MHz}$ to $863 \mathrm{MHz}$ over $3 \mathrm{~V}$ limited by the parasitics of the test setup.

\section{CONCLUSION}

A prototype RF VCO has been built using an on-chip, ICcompatible, high-Q, 3-D coil inductor and micromachined variable capacitor. These passive components match or exceed the performance of discrete counterparts. They are also amenable to monolithic integration with standard IC processes. The oscillator meets the stringent GSM phase-noise requirement and demonstrates that a complete monolithic high-performance VCO can be achieved for cellular telephony applications.

\section{ACKNOWLEDGMENTS}

The authors wish to thank Christopher Harvey, Leland Evans, and Robert T. Graff from Lawrence Livermore National Laboratory for the processing assistance. Certain fabrication steps were carried out in the Berkeley Microfabrication Labora- tory. Phase-noise testing was performed at Philips Semiconductors.

\section{REFERENCES}

[1] P. Davis, et al., "Silicon-on-Silicon Integration of a GSM Transceiver with VCO Resonator", ISSCC Dig. Tech. Papers, pp.248-249, February 1998.

[2] P. R. Gray, R. G. Meyer, "Future Direction in Silicon ICs for RF Personal Communications”, CICC Dig. Tech. Papers, pp. 83-90, May 1995.

[3] B. Razavi, "A 1.8GHz CMOS Voltage-Controlled Oscillator”, ISSCC Dig. Tech. Papers, pp. 388-389, February 1997.

[4] M. Zannoth, B. Kolb, J. Fenk, and R. Weigel, "A Fully Integrated VCO at 2GHz”, ISSCC Dig. Tech. Papers, pp. 224-225, February 1998.

[5] J. N. Burghartz, et al., "Microwave Inductors and Capacitors in Standard Multilevel Interconnect Silicon Technology," IEEE Transactions on Microwave Theory and Techniques. vol. 44, no. 1, pp. 100-104, January 1996.

[6] J. Y-C. Chang, A. A. Abidi, and M. Gaitan, "Large Suspended Inductors on Silicon and Their Use in a $2-\mu \mathrm{m}$ CMOS RF Amplifier," IEEE Transactions on Electron Device Letters, vol. 14, no. 5, pp. 246-248, May 1993.

[7] J. N. Burghartz, et al., "Monolithic Spiral Inductors Fabricated Using a VLSI Cu-Damascene Interconnect Technology and Low-Loss Substrate," IEDM Dig. Tech. Papers, pp. 96-99, December 1996.

[8] D. J. Young, V. Malba, J. J. Ou, A. F. Bernhardt, and B. E. Boser, "Monolithic High-Performance Three-Dimensional Coil Inductors for Wireless Communication Applications," IEDM Dig. Tech. Papers, pp. 67-70, December. 1997.

[9] D. J. Young and B. E. Boser, "A Micromachined Variable Capacitor For Monolithic Low-Noise VCOS," Solid-State Sensor and Actuator Workshop, Dig. Tech. Papers, pp. 86-89, June 1996.

[10] L. -S. Fan, S. J. Woodman, R. C. Moore, L. Crawforth, T. C. Reiley, and M. A. Moser, "Batch-Fabricated Area-Efficient Milli-Actuators," Solid-State Sensor and Actuator Workshop, Dig. Tech. Papers, pp. 38-42, June 1994. 